\title{
On the Complexity of Preferred Semantics in Argumentation Frameworks with Bounded Cycle Length
}

\author{
Wolfgang Dvořák, Matthias König, Stefan Woltran \\ TU Wien, Institute of Logic and Computation \\ \{dvorak, mkoenig, woltran\}@dbai.tuwien.ac.at
}

\begin{abstract}
Argumentation frameworks are a core formalism in the field of formal argumentation, with several semantics being proposed in the literature. Among them, preferred semantics is one of the most popular but comes with relatively high complexity. In fact, deciding whether an argument is skeptically accepted, i.e. contained in each preferred extension, is $\Pi_{2}^{\mathrm{P}}$-complete. In this work we study the complexity of this problem w.r.t. the length of the cycles in the considered AF. Our results show which bounds are necessary to decrease the complexity to coNP and $\mathrm{P}$, respectively. We also consider argumentation frameworks with collective attacks and achieve $\Pi_{2}^{\mathrm{P}}$-hardness already for cycles of length 4 .
\end{abstract}

\section{Introduction}

Argumentation is needed to resolve conflicts in potentially inconsistent or incomplete knowledge, which is essential to draw conclusions of any kind. Argumentation frameworks (AFs), introduced in his influential paper by Dung (1995), turned out to be a versatile system for reasoning tasks in an intuitive setting. In AFs we view arguments just as abstract entities, represented by nodes in a directed graph, independent of their internal structure. Conflicts are modelled in form of attacks between these arguments, constituting the edges of said graph representation. However, by their limited syntax it is hard to formalize certain naturally occurring statements in AFs, which is why various generalizations of the standard formalism have been proposed.

One such generalization extends the syntax by collective attacks (Nielsen and Parsons 2006), i.e. a construction where a set of arguments $T$ attacks an argument $h$, but no proper subset of $T$ does; the resulting class of frameworks is often referred to as SETAFs. The underlying structure of SETAFs then is a directed hypergraph. One of the most popular semantics for AFs and SETAFs is preferred semantics. A preferred extension is a subset-maximal set among the sets of arguments that are conflict-free and defend themselves.

It turned out to be $\Pi_{2}^{\mathrm{P}}$-hard to decide whether an argument is in every preferred extension (Dunne and Bench-Capon 2002), thus an understanding in which scenarios this complexity drops is essential. Cycles have been identified as a source of complexity, which is emphasized by the fact that the problem is P-complete if the framework in question has no cycles (or even just no cycles of even length), and coNPcomplete if there are no cycles of odd length. These results suggest that both even- and odd length cycles contribute to the computational complexity, but in particular a combination of both seem to cause this effect.

The effect of cycles on AFs has been studied in various contexts, e.g. the special issue on loops in AFs (Baroni, Gabbay, and Giacomin 2016), or the concept of weak admissibility (Baumann, Brewka, and Ulbricht 2020; Dauphin, Rienstra, and van der Torre 2020). Regarding computational complexity, previous work has been done on cycle rank (Dvořák, Pichler, and Woltran 2012), even-cyclefreeness (Dunne and Bench-Capon 2001), and odd-cyclefreeness (Dung 1995; Dvořák et al. 2014).

In this work we focus on the complexity of preferred semantics for AFs and SETAFs with bounded cycle length. Our main contributions can be summarized as follows:

- A detailed complexity analysis for the skeptical acceptance problem for a maximal cycle length of $k$ with $0 \leq$ $k \leq \infty$. We provide the exact complexity in most cases, otherwise we state lower and upper bounds.

- For SETAFs, we give a more fine-grained picture and investigate three notions of cycle length for generalizing this concept from graphs to hypergraphs in different ways.

- The extended syntax of SETAFs allows us to pinpoint $\Pi_{2}^{P}$ hardness already for cycles of length 4 (for AFs this case remains open); on the other hand for AFs and a particular notion of cycle length for SETAFs we show coNPmembership for cycles of length 3 .

\section{Preliminaries}

In the following we will introduce the basic notions of argumentation frameworks and complexity classes. First, we recall the basic definitions for AFs (Dung 1995) and SETAFs (Nielsen and Parsons 2006).

Definition 1. An $A F$ is a pair $F=(A, R)$ where $A$ is a non-empty and finite set of arguments, and $R \subseteq A \times A$ is the attack relation.

Definition 2. A SETAF is a pair $S F=(A, R)$ where $A$ is a non-empty and finite set of arguments, and $R \subseteq\left(2^{A} \backslash\{\emptyset\}\right) \times$ $A$ is the attack relation. For an attack $(T, h) \in R$ we call $T$ the tail and $h$ the head of the attack. 
We will consider SETAFs where every attack $(T, h)$ has $|T|=1$ as an AF. We thus define the following notions in terms of SETAFs, subsuming the respective notions for AFs.

Definition 3. Given a SETAF $(A, R)$, we write $S \mapsto_{R} b$ if there is a set $S^{\prime} \subseteq S$ with $\left(S^{\prime}, b\right) \in R$. Moreover, we write $S^{\prime} \mapsto_{R} S$ if $S^{\prime} \mapsto_{R}$ b for some $b \in S$. We drop subscript $R$ in $\mapsto_{R}$ if there is no ambiguity. For $S \subseteq A$, we use $S_{R}^{+}$to denote the set $\left\{b \mid S \mapsto_{R} b\right\}$.

Central for argumentation semantics are the notions of conflict and defense.

Definition 4. Given a SETAF $S F=(A, R)$, a set $S \subseteq A$ is conflicting in $S F$ if $S \mapsto_{R}$ a for some a $\in S$. A set $S \subseteq A$ is conflict-free in $S F$, if $S$ is not conflicting in $S F$, i.e. if $S^{\prime} \cup\{a\} \nsubseteq \subseteq$ for each $\left(S^{\prime}, a\right) \in R$. An argument $a \in A$ is defended (in $S F$ ) by $S \subseteq A$ if for each $B \subseteq A, B \mapsto_{R}$ a implies $S \mapsto_{R} B$. A set $\bar{T}$ of arguments is defended (in $S F$ ) by $S$ if each $a \in T$ is defended by $S$ (in $S F$ ).

Based on these notions a large variety of semantics has been introduced for AFs (Dung 1995; Baroni, Caminada, and Giacomin 2011) and SETAFs (Nielsen and Parsons 2006; Dvořák, Greßler, and Woltran 2018; Flouris and Bikakis 2019). In this work we are interested in preferred semantics, which we introduce next together with admissible and complete sets.

Definition 5. Let $S F=(A, R)$ be a SETAF and let $S \subseteq A$ be a conflict-free set in $S F$. Then, $S$ is admissible (in $S F$ ), if $S$ defends itself in $S F$. $S$ is complete for $S F$, if $S$ is admissible in $S F$ and contains every argument it defends. $S$ is preferred in $S F$, if $S$ is admissible in $S F$ and there is no $T$ admissible in $S F$ such that $T \supset S$.

We recall that every preferred extension is complete.

Complexity. We assume the reader to have basic knowledge in computational complexity theory ${ }^{1}$, in particular we make use of the complexity classes $P$ (polynomial time), coNP, and $\Pi_{2}^{\mathrm{P}}$. For a given SETAF we consider the skeptical acceptance problem Skept $t_{\text {pref }}$ of formal argumentation: Is a given argument contained in all preferred extensions?

The complexity of skeptical acceptance w.r.t. preferred semantics in SETAFs coincides with that of Dung AFs. As SETAFs generalize Dung AFs the hardness results for Dung AFs (Dvořák and Dunne 2017) carry over to SETAFs. Also the same upper bounds hold (Dvořák, Greßler, and Woltran 2018). Hence, in general, Skept $t_{\text {pref }}$ is $\Pi_{2}^{\mathrm{P}}$-complete.

\section{Bounded Cycle Length in AFs}

First, we will define what we mean by maximal cycle length. In the following, a cycle in an AF will be a directed, nonrepeating cycle in the graph structure, the length is given by the number of arguments in the cycle. More formally:

Definition 6. Let $F=(A, R)$ be an $A F$, a cycle $C$ of length $n$ is a sequence $C=\left(a_{1}, a_{2}, \ldots, a_{n}, a_{1}\right)$, such that all $a_{i}$ are distinct, for each $a_{i}$ with $1 \leq i \leq n-1$ there is an

\footnotetext{
${ }^{1}$ For a gentle introduction to complexity theory in the context of formal argumentation, see (Dvořák and Dunne 2017).
}

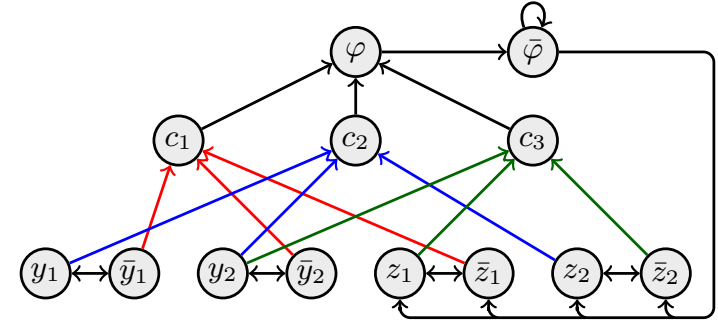

Figure 1: $F^{\Phi}$ for $\Phi=\forall Y \exists Z \varphi(Y, Z)$ with $Y=\left\{y_{1}, y_{2}\right\}, Z=$ $\left\{z_{1}, z_{2}\right\}$, and $\left.\varphi=\left\{\left\{\bar{y}_{1}, \bar{y}_{2}, \bar{z}_{1}\right\},\left\{y_{1}, y_{2}, z_{2}\right)\right\},\left\{y_{2}, z_{1}, \bar{z}_{2}\right\}\right\}$.

attack $\left(a_{i}, a_{i+1}\right) \in R$, and $\left(a_{n}, a_{1}\right) \in R$. The maximal cycle length $m c(F)$ is the length of the longest cycle in $F$, and 0 if $F$ is acyclic.

The following reduction (Dunne and Bench-Capon 2002; Dvořák and Dunne 2017) establishes $\Pi_{2}^{\mathrm{P}}$-hardness for Skept $_{\text {pref }}$ ( $\varphi$ is skeptically accepted in $F^{\Phi}$ iff $\Phi$ is valid).

Reduction 1. Let $\Phi=\forall Y \exists Z C$ be a $Q B F_{\forall}^{2}$-formula consisting of a set of clauses $C$ over sets of propositional atoms $X=Y \cup Z$. We define the $A F F^{\Phi}=(A, R)$, where

$$
\begin{aligned}
A= & \{\varphi, \bar{\varphi}\} \cup C \cup Y \cup \bar{Y} \cup Z \cup \bar{Z}, \\
R= & \{(x, \bar{x}),(\bar{x}, x) \mid x \in X\} \cup \\
& \{(x, c) \mid x \in c\} \cup\{(\bar{x}, c) \mid \bar{x} \in c\} \cup \\
& \{(\varphi, \bar{\varphi}),(\bar{\varphi}, \bar{\varphi})\} \cup\{(\bar{\varphi}, z),(\bar{\varphi}, \bar{z}) \mid z \in Z\}
\end{aligned}
$$

As can be seen in the illustration in Figure 1, this construction has cycles up to a length of 5 . We are interested in whether reasoning becomes easier when we can guarantee that the AF in question does not have any "long" cycles. Indeed, for $\mathrm{AFs} F$ with $m c(F)=0$, i.e. acyclic $\mathrm{AFs}$, reasoning is in $\mathrm{P}$. The problem for AFs with no even length cycles is in $P$ as well (Dunne and Bench-Capon 2001), which settles the case for $m c(F) \leq 1$. In the following, we give an upper bound for the case $2 \leq m c(F) \leq 3$.

Lemma 1. Let $F=(A, R)$ be an AF with $m c(F) \leq 3$ and let $E$ be a complete extension of $F$. Then there is an admissible set $E^{\prime}$ such that $E^{\prime} \supset E$ iff there is an attack $(a, b) \in R$ with $a \notin E$ such that $E \cup\{a\}$ is admissible.

Proof. The " $\Leftarrow$ "-direction is trivial as $E \cup\{a\}$ is such a set $E^{\prime}$. For the " $\Rightarrow$ "-direction assume $E$ is complete and $E^{\prime}$ is admissible with $E^{\prime} \supset E$, i.e. there is an argument $a_{1} \in E^{\prime} \backslash E$. As $E$ is complete, we know $E$ does not defend $a_{1}$, otherwise we would have $a_{1} \in E$. Thus there is at least one attack $\left(b_{1}, a_{1}\right)$ towards $a_{1}$ such that $b_{1} \in E_{R}^{\prime+} \backslash E_{R}^{+}$. We either have that $E \cup\left\{a_{1}\right\}$ defends itself against all of these attacks and thus $E \cup\left\{a_{1}\right\}$ is admissible (and we are done), or there is at least one attack $\left(b_{1}, a_{1}\right)$ such that $E \cup\left\{a_{1}\right\}$ does not attack $b_{1}$. As $E^{\prime}$ is admissible we get that $E^{\prime}$ attacks $b_{1}$, i.e. there is an attack $\left(a_{2}, b_{1}\right)$ with $a_{2} \in E^{\prime} \backslash E$.

Now we can proceed with a similar reasoning as before. We either have that $E \cup\left\{a_{2}\right\}$ defends itself against all attacks on $a_{2}$ and thus $E \cup\left\{a_{2}\right\}$ is admissible, or there is an attack $\left(b_{2}, a_{2}\right)$ such that $E \cup\left\{a_{2}\right\}$ does not attack $b_{2}$. But then there is an argument $a_{3} \in E^{\prime} \backslash E$ that attacks $b_{2}$, and so on. 
By inductively applying this argument we obtain a sequence $\left(a_{1}, b_{1}, a_{2}, b_{2}, \ldots\right)$. If this sequence is finite, for the final argument $a_{f}, E \cup\left\{a_{f}\right\}$ is admissible (otherwise there would be a $b_{f+1}$ ) and thus the claims holds. Towards a contradiction assume the sequence is infinite. As the AF is finite and the $a_{i}$ and $b_{i}$ are from disjoint sets, there is an even length cycle with either $a_{i}=a_{j}$ or $b_{i}=b_{j}$. Notice that in both cases $j \neq i+1$ as we excluded arguments $b_{i}$ such that $a_{i}$ attacks $b_{i}$. But then we have a cycle of length at least 4 , which is in contradiction to our assumption $m c(F) \leq 3$.

In fact, as this proof does not rely on a maximal length of odd length cycles, this argument also applies for AFs with arbitrarily long odd length cycles and no even length cycles of length $\geq 4$. This property yields an algorithm to verify preferred extensions in polynomial time, which in turn means that skeptical acceptance is in coNP.

Lemma 2. Given an $A F F$ with $m c(F) \leq 3$ and a set $S$, deciding whether $S$ is a preferred extension of $F$ is in $P$.

Proof. One can check in polynomial time whether $S$ is complete and for each attack $(a, b)$ whether $a \notin S$ and $S \cup\{a\}$ is admissible: there are only polynomially many attacks and deciding whether a set is admissible is in P. By Lemma 1 this check suffices to decide whether $S$ is preferred.

Lemma 3. Skept ${ }_{\text {pref }}$ for $A F s=(A, R)$ with $m c(F) \leq 3$ is coNP-complete.

Proof. The complementary problem, i.e. deciding whether an argument $a \in A$ is not skeptically accepted, can be decided by guessing a set $S \subseteq A$ with $a \notin A$ and using a $\mathrm{P}$ oracle to verify that $S$ is a preferred extension. Lemma 2 shows that the verification problem for preferred semantics for $m c(F) \leq 3$ is in $\mathrm{P}$. The standard reduction (cf. (Dvořák and Dunne 2017)) has a maximal cycle length of 2 and thus proves coNP-hardness for Skept $t_{\text {pref }}$ for $m c(F) \leq 3$.

For $m c(F) \leq 4$ the case is more complicated. The coNPhardness from $m c(F) \leq 3$ is a lower bound, and the general case provides a $\Pi_{2}^{\mathrm{P}}$ upper bound, but it is not established whether one of these is a tight restriction. The results of this section are summarized in the following theorem.

Theorem 1. For AFs F with bounded cycle length, the complexity results in Table 1 hold. Moreover, if $F$ has no even length cycles of length $\geq 4$, Skept $t_{\text {pref }}$ is coNP-complete.

Lower bounds (i.e. hardness results) carry over from AFs to SETAFs. Upper bounds (i.e. algorithms) for SETAFs also apply for AFs. In the next section we will examine exact complexity results for various SETAF-cycle notions.

\section{Bounded Cycle Length in SETAFs}

Directed cycles in AFs can be generalized to SETAFs in various ways. We will investigate bounded cycle length for skeptical reasoning with the notion of the incidence graph as a starting point, which has applications e.g. in translations from SETAFs to AFs (Polberg 2017). In the incidence graph, the SETAF $(A, R)$ is represented as a bipartite directed graph, with the arguments $A$ as one part, and the tails (a)

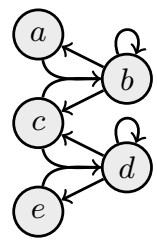

(b)

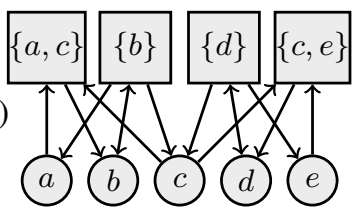

(c)

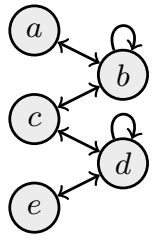

Figure 2: (a) A SETAF $S F$, (b) $\operatorname{Inc}(S F)$, (c) $\operatorname{Primal}(S F)$.

of the attacks in $R$ as the other. We add an edge from every argument to the tails it appears in, and edges from every tail to arguments attacked by it (see Figure 2a \& 2b).

Definition 7. For a SETAF $S F=(A, R)$ let tails $(S F)=$ $\{T \mid(T, h) \in R\}$. Then $\operatorname{Inc}(S F)=(V, E)$ with $V=$ $A \cup$ tails $(S F)$ and $E=\{(t, T),(T, h) \mid(T, h) \in R, t \in T\}$ is its incidence graph.

Definition 8. Let $S F=(A, R)$ be a SETAF. A cycle $C$ of length $n$ is a directed cycle $C=\left(T_{1}, a_{1}, T_{2}, a_{2}, \ldots, a_{n}, T_{1}\right)$ in $\operatorname{Inc}(S F)$. We say $C$ is (i) an incidence-cycle if all $a_{i}$ and all $T_{i}$ are distinct; (ii) a primal-cycle if all $a_{i}$ are distinct; and (iii) $a$ set-cycle if all $T_{i}$ are distinct.

As the name suggests, a primal-cycle corresponds to a cycle in the primal graph (Dvořák, König, and Woltran 2021), a representation of a SETAF as a directed graph. For a SETAF $S F=(A, R)$, its primal graph $\operatorname{Primal}(S F)$ is the directed graph with $A$ as its vertices, and an edge between two vertices $a$ and $b$ iff $a$ is part of an attack towards $b$ in $R$ (for an example see Figure 2). Every incidence-cycle is a primal-cycle and a set-cycle. Moreover, a SETAF is incidence-acyclic iff it is primal-acyclic iff it is set-acyclic. Finally, note that on AFs all of these cycle notions coincide with 'classical' directed, non-repeating cycles. This leads us to our different notions for the (maximal) cycle length.

Definition 9. Given a SETAF $S F$, let $m c^{I}(S F), m c^{P}(S F)$, $m c^{S}(S F)$ be the length of its longest incidence-cycle, primal-cycle, set-cycle, resp., and 0 if $S F$ has no such cycle.

We have that $m c^{I}(S F) \leq \min \left(m c^{P}(S F), m c^{S}(S F)\right)$. In SETAFs $S F$ for $m c^{X}(S F) \leq 1$ with $X \in\{I, P, S\}$ the problem Skept $t_{\text {pref }}$ is P-complete: it is easy to show that - as in AFs (Dunne and Bench-Capon 2001) - every SETAF SF with more than one complete extension has an even length incidence-cycle. Hence, every SETAF without even length incidence-cycles has only one preferred extension.

The same proof we used to establish coNP-membership for AFs $F$ with $m c(F) \leq 3$ works with little adaptations for SETAFs $S F$ with $m c^{S}(S F) \leq 3$. However, for our other cycle notions this does not hold, as the SETAF in Figure 2 illustrates: the complete extension $\emptyset$ cannot be "extended' to an admissible set by adding the tail of any attack in $S F$, even though there is an admissible set $\{a, c, e\} \supset \emptyset$. $(\{a, c\}, b,\{b\}, c,\{c, e\}, d,\{d\}, c,\{a, c\})$ is a set-cycle but neither a primal-cycle nor an incidence-cycle. Hence, we have $m c^{S}(S F)=4$ and $m c^{I}(S F)=m c^{P}(S F)=2$.

Next, we establish $\Pi_{2}^{\mathrm{P}}$-hardness for the problem Skept $t_{\text {pref }}$ in SETAFs $S F$ with $m c^{X}(S F) \leq 4$ and $X \in\{I, P, S\}$. To this end, we introduce a new reduction from the $Q B F_{\forall}^{2}$ problem to Skept $_{\text {pref }}$ with a maximal cycle length of 4 . 


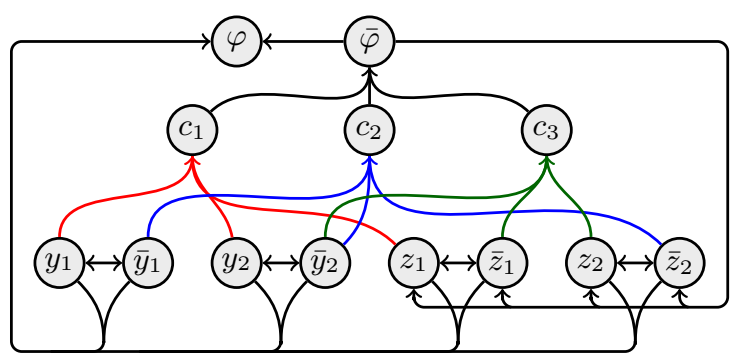

Figure 3: $S F^{\Phi}$ for $\Phi=\forall Y \exists Z \varphi(Y, Z)$ with $Y=\left\{y_{1}, y_{2}\right\}, Z=$ $\left\{z_{1}, z_{2}\right\}$, and $\left.\varphi=\left\{\left\{\bar{y}_{1}, \bar{y}_{2}, \bar{z}_{1}\right\},\left\{y_{1}, y_{2}, z_{2}\right)\right\},\left\{y_{2}, z_{1}, \bar{z}_{2}\right\}\right\}$.

Reduction 2. Let $\Phi=\forall Y \exists Z C$ be a $Q B F_{\forall}^{2}$-formula consisting of a set of clauses $C$ over sets of propositional atoms $X=Y \cup Z$. We define the SETAF $S F^{\Phi}=(A, R)$, where

$$
\begin{aligned}
A= & \{\varphi, \bar{\varphi}\} \cup C \cup Y \cup \bar{Y} \cup Z \cup \bar{Z}, \\
R= & \{(\{x\}, \bar{x}),(\{\bar{x}\}, x) \mid x \in X\} \cup \\
& \{(\{x \mid \bar{x} \in c\} \cup\{\bar{x} \mid x \in c\}, c) \mid c \in C\} \cup \\
& \{(C, \bar{\varphi}),(\{\bar{\varphi}\}, \varphi)\} \cup\{(\{x, \bar{x}\}, \varphi) \mid x \in X\} \cup \\
& \{(\{\bar{\varphi}\}, z),(\{\bar{\varphi}\}, \bar{z}) \mid z \in Z\} .
\end{aligned}
$$

W.l.o.g. we assume every clause of the formula to contain at least one literal from $Z \cup \bar{Z}$. For an illustration see Figure 3. The main difference between this reduction and Reduction 1 is that here a clause is jointly attacked by the duals of the literals in this clause. This way, a clause is attacked only if the corresponding interpretation makes its duals true. If at least one clause is attacked, $\varphi$ cannot be defended. The attacks from the $x_{i}$ and $\bar{x}_{i}$ towards $\varphi$ ensure that every admissible set that contains $\varphi$ also contains either $x$ or $\bar{x}$ (as otherwise $\varphi$ would not be defended). In fact, the longest cycle in $S F^{\Phi}$ is of length 4 .

Lemma 4. $\varphi$ is in every pref. extension of $S F^{\Phi}$ iff $\Phi$ is valid.

Proof. We start with general observations about $S F^{\Phi}$. For each $y \in Y$, every preferred extension either contains $y$ or $\bar{y}$. The arguments in $Z \cup \bar{Z}$ can only be in an admissible set $S$ if $\bar{\varphi}$ is attacked and consequently only if $C \subseteq S$. Note that we assume for the $Q B F_{\forall}^{2}$ formula every clause $c$ to have at least one literal from $Z \cup \bar{Z}$ and thus no admissible set without arguments $z$ or $\bar{z}$ attacks any arguments $c \in C$. That is, no such admissible set can contain $\bar{\varphi}$. On the other hand, we need to attack $\bar{\varphi}$ in order to include arguments $z$ or $\bar{z}$. Hence, there is no admissible set containing $\bar{\varphi}$.

" $\Rightarrow$ ": Every interpretation $\mathcal{I}_{Y}$ over $Y$ corresponds to an admissible set $\left\{y_{i} \mid \mathcal{I}_{Y}\left(y_{i}\right)=\right.$ true $\} \cup\left\{\bar{y}_{i} \mid \mathcal{I}_{Y}\left(y_{i}\right)=\right.$ false $\}$ in $S F^{\Phi}$ and thus there is at least one preferred extension which contains these arguments. Consider one of these preferred extensions $E$. By assumption we have $\varphi \in E$ and thus $\bar{\varphi} \in E_{R}^{+}$, i.e. $C \subseteq E$. We next define $\mathcal{I}_{Z}(z)=$ true if $z \in E$ and $\mathcal{I}_{Z}(z)=$ false otherwise. As $E \cap(Y \cup \bar{Y} \cup Z \cup \bar{Z})$ must defend all $c \in C$ and by the definition of the attack re-

\begin{tabular}{|c|c|c|c|}
\hline$k$ & $m c(F) \leq k$ & $\begin{array}{l}m c^{I}(S F) \leq k \\
m c^{P}(S F) \leq k\end{array}$ & $m c^{S}(S F) \leq k$ \\
\hline$\geq 5$ & $\begin{array}{c}\Pi_{2}^{P}-c \\
c P_{-h}\end{array}$ & $\Pi_{2}^{P}-\mathrm{c}$ & $\begin{array}{l}\Pi_{2}^{\mathrm{P}}-\mathrm{c} \\
\Pi_{-c}\end{array}$ \\
\hline $\begin{array}{c}4 \\
3,2\end{array}$ & $\begin{array}{c}\text { coNP-c } \\
\text { cons }\end{array}$ & coNP-h / in $\Pi^{P}$ & $\begin{array}{l}1 \mathrm{I}_{2}^{-\mathrm{c}} \\
\text { coNP-c }\end{array}$ \\
\hline 1,0 & P-c & $P-c$ & P-c \\
\hline
\end{tabular}
lation we obtain that $\mathcal{I}_{Y} \cup \mathcal{I}_{Z}$ is a satisfying assignment for the formula. We have shown that for every interpretation $\mathcal{I}_{Y}$ there is an interpretation $\mathcal{I}_{Z}$ such that $\mathcal{I}_{Y} \cup \mathcal{I}_{Z}$ satisfies the formula. Hence, the $Q B F_{\forall}^{2}$-formula is valid.
Table 1: Complexity of Skept $t_{\text {pref }}$ for AFs (Col. 1) and SETAFs (Col. 2 and 3); $\mathcal{C}$-c denotes completeness for the complexity class $\mathcal{C}$.

" $\Leftarrow$ ": Towards a contradiction assume that there is a preferred extension $E$ with $\varphi \notin E$. We can define $\mathcal{I}_{Y}$ such that $\mathcal{I}_{Y}(y)=$ true if $y \in E$ and $\mathcal{I}_{Y}(y)=$ false otherwise. Now as $\Phi$ is valid, for every partial assignment $\mathcal{I}_{Y}$ there is a partial assignment $\mathcal{I}_{Z}$ such that $\mathcal{I}_{Y} \cup \mathcal{I}_{Z}$ make $\varphi$ true. That is, by the arguments in first part, there is a preferred extension $E^{\prime}=\{\varphi\} \cup\left\{y_{i} \mid \mathcal{I}_{Y}\left(y_{i}\right)=\right.$ true $\} \cup\left\{\bar{y}_{i} \mid \mathcal{I}_{Y}\left(y_{i}\right)=\right.$ false $\} \cup\left\{z_{i} \mid \mathcal{I}_{Z}\left(z_{i}\right)=\right.$ true $\} \cup\left\{\bar{z}_{i} \mid \mathcal{I}_{Z}\left(z_{i}\right)=\right.$ false $\} \cup C$. We next argue that $E \subset E^{\prime}$. By construction $E$ and $E^{\prime}$ coincide on $Y$ an $\bar{Y}$. Moreover, $C \cap E \neq C$, as otherwise we would have either $z_{i} \in E$ or $\bar{z}_{i} \in E$ for each $z_{i}$ and $\varphi$ defended, i.e. $\varphi \in E$, a contradiction. Consequently, $E \cap(Z \cup \bar{Z})=\emptyset$. Hence, $E \subset E^{\prime}$, which is in contradiction to $E$ being a preferred extension.

Finally, these results let us state the exact complexity of skeptical reasoning in preferred semantics for SETAFs of bounded cycle length. Hardness results carry over from AFs to SETAFs, as all three cycle notions coincide with classical non-repeating directed cycles in the special case of AFs.

Theorem 2. For SETAFs $S F$ with bounded cycle length, the complexity results in Table 1 hold.

\section{Conclusion}

In this work we investigated the effect of a bounded cycle length on the complexity of skeptical acceptance w.r.t. preferred semantics. We showed that for AFs and SETAFs (when considering the set-cycle notion) where the length of cycles is bounded by 3 (or the length of even length cycles is bounded by 2), we can iteratively construct preferred extension from admissible sets. Therefore, the complexity of Skept $t_{\text {pref }}$ drops to the first level of the polynomial hierarchy. While in AFs especially odd length cycles contribute largely to the hardness of skeptical acceptance, in SETAFs some odd length cycles can be expressed in terms of even length cycles where 'traditional' single-argument-attacks are replaced by collective attacks - the only way to achieve conjunctive behaviour (i.e. an argument is attacked iff all of some other arguments are attacked) is in terms of defense, i.e. by introducing an additional argument that has to be defended against all incoming attacks (in Reduction 1 this argument is $\varphi$ ). While we provided lower and upper bounds in most cases, there are still some gaps in the complexity landscape of AFs and SETAFs w.r.t. bounded cycle length. Besides closing these gaps, future work includes investigations of other semantics. For this, the results from (König 2020) can serve as a starting point. 


\section{Acknowledgments}

This research has been supported by the Vienna Science and Technology Fund (WWTF) through project ICT19-065, and by the Austrian Science Fund (FWF) through projects P30168, P32830, and Y698.

\section{References}

Baroni, P.; Caminada, M.; and Giacomin, M. 2011. An introduction to argumentation semantics. Knowledge Eng. Review 26(4):365-410.

Baroni, P.; Gabbay, D. M.; and Giacomin, M., eds. 2016. Special Issue on Loops in Argumentation, volume 26(4) of J. Log. Comput. Oxford University Press.

Baumann, R.; Brewka, G.; and Ulbricht, M. 2020. Revisiting the foundations of abstract argumentation - semantics based on weak admissibility and weak defense. In The Thirty-Fourth AAAI Conference on Artificial Intelligence, AAAI 2020, The Thirty-Second Innovative Applications of Artificial Intelligence Conference, IAAI 2020, The Tenth AAAI Symposium on Educational Advances in Artificial Intelligence, EAAI 2020, New York, NY, USA, February 7-12, 2020, 2742-2749. AAAI Press.

Dauphin, J.; Rienstra, T.; and van der Torre, L. 2020. A principle-based analysis of weakly admissible semantics. In Prakken, H.; Bistarelli, S.; Santini, F.; and Taticchi, C., eds., Computational Models of Argument - Proceedings of COMMA 2020, Perugia, Italy, September 4-11, 2020, volume 326 of Frontiers in Artificial Intelligence and Applications, 167-178. IOS Press.

Dung, P. M. 1995. On the acceptability of arguments and its fundamental role in nonmonotonic reasoning, logic programming and n-person games. Artif. Intell. 77(2):321-358.

Dunne, P. E., and Bench-Capon, T. J. M. 2001. Complexity and combinatorial properties of argument systems. Technical report, Dept. of Computer Science, University of Liverpool.

Dunne, P. E., and Bench-Capon, T. J. M. 2002. Coherence in finite argument systems. Artif. Intell. 141(1/2):187-203.

Dvořák, W.; König, M.; and Woltran, S. 2021. Graphclasses of argumentation frameworks with collective attacks. In Faber, W.; Friedrich, G.; Gebser, M.; and Morak, M., eds., Logics in Artificial Intelligence - 17th European Conference, JELIA 2021, Virtual Event, May 17-20, 2021, Proceedings, volume 12678 of Lecture Notes in Computer Science, 3-17. Springer.

Dvořák, W., and Dunne, P. E. 2017. Computational problems in formal argumentation and their complexity. FLAP 4(8):2557-2622.

Dvořák, W.; Järvisalo, M.; Wallner, J. P.; and Woltran, S. 2014. Complexity-sensitive decision procedures for abstract argumentation. Artif. Intell. 206:53 - 78.

Dvořák, W.; Greßler, A.; and Woltran, S. 2018. Evaluating SETAFs via answer-set programming. In Thimm, M.; Cerutti, F.; and Vallati, M., eds., Proceedings of the Second International Workshop on Systems and Algorithms for Formal Argumentation (SAFA 2018) co-located with the 7th
International Conference on Computational Models of Argument (COMMA 2018), Warsaw, Poland, September 11, 2018, volume 2171 of CEUR Workshop Proceedings, 1021. CEUR-WS.org.

Dvořák, W.; Pichler, R.; and Woltran, S. 2012. Towards fixed-parameter tractable algorithms for abstract argumentation. Artif. Intell. 186:1 - 37.

Flouris, G., and Bikakis, A. 2019. A comprehensive study of argumentation frameworks with sets of attacking arguments. Int. J. Approx. Reason. 109:55-86.

König, M. 2020. Graph-classes of argumentation frameworks with collective attacks. Master's thesis, TU Wien.

Nielsen, S. H., and Parsons, S. 2006. A generalization of Dung's abstract framework for argumentation: Arguing with sets of attacking arguments. In Maudet, N.; Parsons, S.; and Rahwan, I., eds., Argumentation in Multi-Agent Systems, Third International Workshop, ArgMAS 2006, Hakodate, Japan, May 8, 2006, Revised Selected and Invited Papers, volume 4766 of Lecture Notes in Computer Science, 54-73. Springer.

Polberg, S. 2017. Developing the Abstract Dialectical Framework. Ph.D. Dissertation, Vienna University of Technology, Institute of Information Systems. 